\title{
DEVELOPMENT OF IMMERSION SUBUNIT VACCINE AGAINST GROUPER IRIDOVIRUS (GIV)
}

\author{
Hong-Jen Liang ${ }^{1}$, Ren-Hong Peng ${ }^{2}$, and Pinwen P Chiou ${ }^{2,3,8}$ \\ ${ }^{l}$ Department of Food Science, Yuanpei University of Medical Technology, Hsinchu 30015, TAIWAN \\ ${ }^{2}$ Department of Aquaculture, National Taiwan Ocean University, Keelung 20224, TAIWAN \\ ${ }^{3}$ Center of Excellence for the Oceans, National Taiwan Ocean University, Keelung 20224, TAIWAN
}

\begin{abstract}
Iridovirus is one of the most devastating viral pathogens in groupers (Epinephelus spp). The viral infection could often lead to near $100 \%$ mortality in hatcheries, thus a bottleneck to the development of the grouper aquaculture industry. Vaccination is an important and efficient strategy against viral infection. The most common delivery method for commercially available fish vaccines is injection, which is both time-consuming and labor intensive despite the high efficacy. We report here the development of a new adjuvant NE and its application in an immersion GIV subunit vaccine. The adjuvant NE can form low viscosity nanoparticles with average size of about $168 \mathrm{~nm}$ and are safe to grouper. When co-delivered with NE, green fluorescent protein (GFP) can be detected in gills and the digestive tract at 20 min postimmersion. Subsequently, an immersion subunit vaccine was generated by combining NE with recombinant major capsid protein (MCP) of GIV. After vaccination by immersion, MCP-specific antibodies could be detected at 2 weeks post-vaccination. Furthermore, the subunit vaccine enhanced the survival rate of infected fish in a dose-dependent manner. We further evaluated the vaccination protocol of this subunit vaccine. The results showed that boosters (at 1 or 2 weeks after the initial vaccination) enhanced the yield of specific antibodies and the protection against GIV in the vaccinated grouper at both 3 and 4 weeks after initial vaccination. Booster at 1 week had a greater impact than booster at 2 week did. At 10 weeks after initial vaccination, the fish were resistant to GIV in all groups; however, the viral loads in the booster groups was lower than those in the single-vaccination and control groups. In conclusion, out study indicates the potential application of this immersion subunit GIV vaccine as an effective and easy-to-handle vaccine for the grouper aquaculture industry.
\end{abstract}

\section{KEYWORDS}

grouper, iridovirus, major capsid protein, immersion, subunit vaccine

${ }^{\S}$ Corresponding author. Tel.: +886 224622192 ext 5215; Fax: +886 224633150.

E-mail address: ppchiou@mail.ntou.edu.tw 